\title{
Comparison of Different Sperm Selection Techniques in Ram Frozen-Thawed Sperm
}

\author{
Carolina Cerqueira Sarmento Olivares', Joanna Maria Gonçalves de Souza-Fabjan, 1,2, \\ Jeferson Ferreira da Fonseca ${ }^{3}$, Mario Felipe Alvarez Balaro', Vicente José de Figueirêdo Freitas ${ }^{4}$, \\ Rodrigo Vasconcelos de Oliveira ${ }^{5}$ \& Felipe Zandonadi Brandão ${ }^{1}$
}

\begin{abstract}
Background: The success of fertilization is directly associated with semen quality and the sperm preparation. Considering the common use of cryopreserved spermatozoa, there is a need to develop strategies for sperm preparation in order to achieve a sperm sample of high quality through a rigorous selection of sperm. Thus, sperm cells are being more extensively investigate. This study aimed evaluating the influence of different sperm selection techniques on ram sperm parameters in semen preparation.

Materials, Methods \& Results: Frozen-thawed commercial semen from 10 Santa Inês rams was subjected to either: swim-up, Percoll, mini-Percoll, sperm washing by centrifugation or a control group. After each technique, samples were incubated at $37^{\circ} \mathrm{C}$ for $1 \mathrm{~h}, 2 \mathrm{~h}$ and $3 \mathrm{~h}$. At post-selection moment $(0 \mathrm{~h})$ and at each interval, sperm recovery rate, motility, capacitation and plasma membrane $(\mathrm{PM})$ integrity were analyzed. The lowest $(P<0.05)$ recovery rate was recorded after swim-up $(1.0 \pm 0.3 \%)$, whilst the others were similar $(P>0.05)$. Most part of motility parameters were not affected $(P>0.05)$ by the technique at $0 \mathrm{~h}$; just swim-up obtained higher $(P<0.05)$ values for VSL $(41.8 \pm 11.1)$ and VAP $(46.9$ $\pm 11.2)$. Overall, swim-up presented higher $(P<0.05)$ values for most of motility parameters over time of incubation. The control group led to more $(P<0.05)$ capacitated cells $(46.8 \pm 2.3 \%)$, whilst Percoll, mini-Percoll and swim-up were similar $(\sim 33 \% ; P>0.05)$, regardless of interval incubation. However, the latter three techniques presented more $(P<0.05)$ reacted cells. Swim-up recovered a higher $(P<0.05)$ number of intact cells $(32.1 \pm 6.4 \%)$, and Percoll, mini-Percoll and control group were similar $(P>0.05)$.

Discussion: The present study evaluated the sperm cell and its preparation for receiving the oocyte under optimal conditions. evaluate the sperm cell and its preparation for receiving the oocyte under optimal conditions. Although the swim-up technique promoted higher rates for some of the sperm parameters evaluated, Percoll protocols are the most widely used procedures for selection during the sperm preparation in many species, possibly because of its greater speed, practical method and convenience compared to the swim-up technique. Percoll and mini-Percoll recovered approximately 10 times more cells than swim-up, which is an important feature to be considered during sperm preparation for in vitro fertilization (IVF), being possible to use only one semen straw. The high capacitated and acrosome reaction rates observed after the treatments in the current study, are probably reinforced by changes in sperm cells caused by the cryopreservation process. In order to strength the evidence that frozen-thawed sperm, even after selection, is sensible and reactive to capacitation-like events, we demonstrated the capacitated and acrosome-reacted cell values immediately after the selection treatments behaved differently than when authors used ram fresh sperm. Possibly, this capacitation-like changes observed in frozen-thawed sperm occurs regardless of the selection treatment used. Analyzing the motility parameters immediately after the selection, all treatments maintained or increased the rates compared to the control group. The swim-up, mini-Percoll and Percoll did not differ in any parameters. Given that Percoll and mini-Percoll did not show differences in relation to swim-up for motility parameters, such techniques can be used to replace the latter, obtaining similar sperm samples with good quality. However, swim-up technique involves a procedure that recovers a clean fraction without debris and other types of cells, with high rate of mobile sperm with excellent quality, reason why it can justify the higher recovery of intact spermatozoa after the technique.
\end{abstract}

Keywords: ovine, sperm preparation, sperm capacitation, CASA, CTC, plasma membrane integrity. 


\section{INTRODUCTION}

The ability to assess the fertilizing potential of a sperm sample is still considered a very difficult task and, unfortunately, there is not a single definitive and effective test that can predict such capacity. Thus, sperm cells are being more extensively investigated and their importance has been emphasized [32]. Considering the common use of cryopreserved spermatozoa, there is a need to develop strategies for sperm preparation in order to achieve a sperm sample of high quality through a rigorous selection of sperm [39]. To be effective, the sperm preparation method aims the selection of high quality sperm, with good rates of motility and viability [33].

In vivo, the sperm selection process is controlled by the female reproductive tract to ensure that the descendants receive high quality genetic material from the male gamete [11]. In order to achieve a sperm selection based on natural principles, researchers need to understand these events and reproduce them [21], through the development of a sperm preparation of excellence.

The sperm selection methods used in sheep are: swim-up [15,34], Percoll density gradients $[9,38]$ and sperm washing by centrifugation $[4,18]$. The use of mini-Percoll technique appears as an attractive alternative to minimize the cost and reduce the contact with the density gradient molecules. Thus, the aim of the present study was to compare the efficacy of different sperm selection techniques: (i) Percoll density gradient; (ii) mini-Percoll density gradient; (iii) swim-up; (iv) sperm washing by centrifugation, on the recovery of high-quality frozen-thawed ram spermatozoa for further assisted reproductive biotechnologies.

\section{MATERIALS AND METHODS}

All the experiments were carried out at Fluminense Federal University (UFF), Niterói (Brazil, latitude $22^{\circ} 53^{\prime} \mathrm{S}$, longitude $43^{\circ} 06^{\prime} \mathrm{W}$ ). The procedures were approved by the local ethic committee.

\section{Reagents}

All chemicals were from Sigma Chemical ${ }^{1}$. Exceptions were acridine orange and propidium iodide of VitalTest kit obtained from Halotech DNA ${ }^{2}$.

\section{Experimental design}

Commercial semen straws $(0.25 \mathrm{~mL})$ of the same batch from 10 Santa Inês rams aging 2-5 years old, sexually matured and of proved fertility were used. All straws were maintained frozen in liquid nitrogen until their use. The straws were thawed at $37^{\circ} \mathrm{C}$ for $30 \mathrm{~s}$. A pool of five straws of the same ram was thawed and homogenized in a warmed $1.5 \mathrm{~mL}$ microtube ${ }^{3}$. At post-thawing (PT), a sample of the pool was removed to evaluate rates of sperm concentration and motility. Then, the semen was divided into $0.15 \mathrm{~mL}$ aliquots and each one was submitted to either: Percoll density gradient, mini-Percoll density gradient, swim-up or sperm washing by centrifugation. At the same moment, another $0.15 \mathrm{~mL}$ aliquot was also transferred to a 300 $\mathrm{x} g$ centrifugation for $8 \mathrm{~min}$, in a $15 \mathrm{~mL}$ conical tube containing $4 \mathrm{~mL}$ SP-TALP (supplemented with $3 \mathrm{mg}$ / mL BSA V, $2.2 \mathrm{mg} / \mathrm{mL}$ sodium pyruvate, 50,000UI/ $\mathrm{mL}$ penicillin and $50 \mathrm{mg} / \mathrm{mL}$ streptomycin), featuring the control group, free of any sperm selection technique influence. At the end of all treatments, aliquots (post-selection $=0 \mathrm{~h}$ ) were taken to quantify the rates of sperm recovery, motility, capacitation and plasma membrane (PM) integrity. Afterwards, samples of all treatments were submitted to incubation at $37^{\circ} \mathrm{C}$, for 1 $\mathrm{h}, 2 \mathrm{~h}$ and $3 \mathrm{~h}$ and the same parameters mentioned were assessed among the intervals, except the recovery rate.

\section{Traditional Percoll technique}

The sperm selection by Percoll technique was established based on the method described by Papadopoulos et al. [26], with slight modifications. A total of $2 \mathrm{~mL}$-gradient (90/45\% density) of Percoll solution was prepared in a $15 \mathrm{~mL}$ conical centrifuge tube ${ }^{4}$. A $0.15 \mathrm{~mL}$ aliquot of semen described above was poured into the Percoll gradient tube and it was subjected to a $700 \times \mathrm{g}$ centrifugation for $10 \mathrm{~min}$. At the end of this centrifugation, an aliquot was removed to assess the motility and the sample was subjected to another centrifugation at $200 \mathrm{x} g$ for $5 \mathrm{~min}$. After the second centrifugation, the supernatant was discarded and the pellet was resuspended in $0.25 \mathrm{~mL}$ of SP-TALP.

\section{Mini-Percoll technique}

The gradient was formed by pipetting 0.4 $\mathrm{mL}$ of $90 \%$ Percoll solution into a $2 \mathrm{~mL}$ microtube and then overlaying it with $0.4 \mathrm{~mL}$ of $45 \%$ Percoll solution. A $0.15 \mathrm{~mL}$ aliquot of the pool was placed onto the top of the $45 \%$ layer and then centrifuged twice by $5000 \times g$ for $5 \mathrm{~min}$ [16]. At the end of the centrifugation, the supernatant was removed and the pellet was suspended in $0.4 \mathrm{~mL}$ of SP-TALP, 
and then, the sample was submitted to another centrifugation. After last centrifugation, the supernatant was removed and the pellet was suspended in 0.25 $\mathrm{mL}$ of SP-TALP.

\section{Swim-up}

Swim-up procedure was previously described by Morton et al. [23], but some modifications were performed in the present study. A $0.15 \mathrm{~mL}$ aliquot of the pool of thawed sperm was carefully placed in the bottom of a $15 \mathrm{~mL}$ conical tube containing $1 \mathrm{~mL}$ SPTALP, supplemented with $3 \mathrm{mg} / \mathrm{mL}$ BSA V, $2.2 \mathrm{mg} /$ $\mathrm{mL}$ sodium pyruvate, 50,000 IU $\mathrm{mL}$ penicillin and $50 \mathrm{mg} / \mathrm{mL}$ streptomycin. Sperm cells were allowed to swim-up in a $45^{\circ}$ position for $45 \mathrm{~min}$, at $38.5^{\circ} \mathrm{C}$ in a humidified $5 \% \mathrm{CO}_{2}$ (in air) atmosphere. After this incubation, the supernatant with the spermatozoa was harvested and the motility was assessed. Then, the spermatozoa were transferred to another tube containing $1 \mathrm{~mL}$ SP-TALP and centrifuged at $300 \times \mathrm{x}$ for 8 min. Finally, the pellet was resuspended in $0.25 \mathrm{~mL}$ of SP-TALP.

\section{Sperm washing by centrifugation}

This technique was performed following the method described by Mara et al. [18], with some modifications. A $0.15 \mathrm{~mL}$ aliquot of the thawed sperm pool was placed in a $15 \mathrm{~mL}$ conical tube containing 6 $\mathrm{mL}$ of SP-TALP and washed twice by centrifugation at $200 \mathrm{x} g$ for $5 \mathrm{~min}$. Before the second centrifugation, the pellet was suspended with $6 \mathrm{~mL}$ of SP-TALP and an aliquot was pipetted to assess the motility. After the last centrifugation, the supernatant was discarded and the spermatozoa pellet was resuspended in 0.25 $\mathrm{mL}$ of SP-TALP.

\section{Sperm recovery rate}

This rate was obtained after each treatment by the use of a Neubauer counting chamber after spermatozoa dilution (1:200). The rate was calculated by the formula: [final concentration $\times$ final volume] $\times$ [initial concentration $\times$ initial volume] $-1 \times 100$.

\section{Sperm Capacitation}

The capacitation status was assessed by chlortetracycline (CTC) test and was based at the technique described by Pérez-Pé et al. [29], with slight modifications. A $0.75 \mathrm{mM}$ CTC solution was daily prepared in a buffer containing $20 \mathrm{mM}$ Tris, 130 $\mathrm{mM} \mathrm{NaCl}$ and $5 \mathrm{Mm}$ cysteine, $\mathrm{pH}$ 7.8. For staining,
$0.01 \mathrm{~mL}$ of sperm sample were mixed with $0.01 \mathrm{~mL}$ of CTC solution onto a glass slide. Finally, a drop of an antifading solution (0.22 M 1,4-diazabicyclo [2.2.2] octane, DABCO, Sigma) was mixed to retard the fading of CTC fluorescence. For the evaluation of CTC patterns, the samples were observed in a microscope (Nikon Eclipse $\mathrm{Ci}^{5}$ ) under epifluorescence illumination, in 1000x magnification with oil immersion. One hundred spermatozoa per slide were scored [3] and classified into three groups, as described by Cormier et al. [5]: bright fluorescence over the whole head (noncapacitated cells, F pattern); fluorescence-free band in the postacrosomal region (capacitated cells, B pattern); and full fluorescence over the whole head except for a thin, bright band of fluorescence along the equatorial region (acrosome-reacted cells, AR pattern).

\section{Sperm motility}

Sperm motility parameters were analyzed by computer-assisted semen analysis (CASA) using the $\mathrm{SCA}^{\circledR}$ system (Sperm Class Analyzer Microptic ${ }^{6}$ ), connected to a microscope and the following settings were applied: image collection speed: 25 images per second; magnifying power of microscope: x100; measurements were performed in a 24 x $24 \mathrm{~mm}$ coverslide with a $10 \mu \mathrm{L}$ sample drop volume. Sperm movement was observed under negative phase contrast optics and videotape recorded in three different fields, at least, or 500 spermatozoa were captured for analyzing. Software settings were adjusted to ram spermatozoa. The main software settings were as follows: the sperm head dimensions detected by the system were between 18 and $60 \mu \mathrm{m}^{2}$. Spermatozoa were identified as immotile by curvilinear velocity (VCL) below $10 \mu \mathrm{m} / \mathrm{s}$; between 10 and $45 \mu \mathrm{m} / \mathrm{s}$ were classified as slow; between 45 and $75 \mu \mathrm{m} / \mathrm{s}$ were classified as medium; and above $75 \mu \mathrm{m} / \mathrm{s}$ were classified as rapid. The sperm cells presenting straightness (STR) above $80 \%$ were featured as progressive. The following motility patterns were analyzed: total motility $(\%)$, progressive motility $(\%)$, fast progressive $(\%)$, fast sperm $(\%)$, average path velocity (VAP; $\mathrm{mm} / \mathrm{s})$, VCL $(\mathrm{mm} / \mathrm{s})$, straight line velocity (VSL; $\mathrm{mm} / \mathrm{s}$ ), straightness (STR: VSL/VAP; \%), linearity (LIN: VSL/ VCL; \%), wobble (WOB: VAP/ VCL; $\%)$, lateral head displacement (ALH; mm) and beat cross frequency (BCF; Hz). 


\section{PM integrity}

This assessment was carried out by VitalTest kit and followed the manufacturer instructions. At least 100 spermatozoa per slide were analyzed. The test was performed by CASA system connected to a microscope under epifluorescence illumination, using 100x magnification. This procedure resulted in selective labelling of either damage or normal cells visualized in red or green colors, respectively.

\section{Statistical analysis}

All quantitative variables were subjected to normality (Lilliefors test) and homoscedasticity tests and one-way analysis of variance (ANOVA) was performed with Tukey or Fisher-LSD tests for means comparisons. The non-normal variables were subjected to Kruskal-Wallis test followed by Dunn's test. A value of $P<0.05$ was considered to be statistically significant. Statistical analyses were performed using the $9.0 \mathrm{SAEG}^{\circledR}$ program ${ }^{7}$.

\section{RESULTS}

Sperm recovery rate was similar $(P>0.05)$ among sperm washing, Percoll and mini-Percoll (7.4 \pm 2.3\%; average of these techniques). Swim-up resulted in the lowest $(1.0 \pm 0.3 \% ; P<0.05)$ whilst the control group, in the highest $(31.2 \pm 4.6 \% ; P<0.05)$ rate. The majority of sperm motility parameters assessed were not affected $(P>0.05)$ by the technique, immediately after the selection $(0 \mathrm{~h})$. The only exceptions were VSL, VAP and ALH parameters, as shown in Table 1.

When we compared the intervals of incubation among the treatments on motility parameters, swimup obtained higher $(P<0.05)$ values at most intervals assessed (Table 2). On the other hand, there was no difference $(P>0.05)$ among the incubation intervals after each selection technique for all parameters studied, exception for BCF.

Overall, when we pool the data regardless of the interval of incubation (average from 0 to $3 \mathrm{~h}$ ), there was difference $(P>0.05)$ among the treatments for the majority of the motility parameters assessed. For total motility, swim-up $(32.4 \pm 2.2 \%)$, mini-Percoll (28.1 \pm $2.9 \%)$, Percoll $(28.0 \pm 2.2 \%)$ and control group (28.0 $\pm 3.0 \%)$ did not differ $(P>0.05)$, but swim-up was higher $(P<0.05)$ than sperm washing $(24.6 \pm 2.1 \%)$. For progressive sperm, swim-up $(9.3 \pm 1.6 \%)$, miniPercoll $(6.0 \pm 1.9 \%)$ and Percoll $(4.5 \pm 1.2 \%)$ were not different $(P>0.05)$ among each other, but swim-up was higher $(P<0.05)$ than sperm washing $(3.6 \pm 1.1 \%)$ and control group $(3.9 \pm 1.3 \%)$. Swim-up presented the highest value for fast sperm $(7.2 \pm 1.4 \% ; P<0.05)$, but it did not differ $(P>0.05)$ to mini-Percoll $(4.6 \pm 1.5 \%)$.

Mini-Percoll and Percoll were similar $(P>$ 0.05 ), regardless of the interval of incubation (average from 0 to $3 \mathrm{~h}$ ), for all velocities studied (VCL: $40.1 \pm$ 4.6 vs. $39.1 \pm 5.1$; VSL: $20.1 \pm 3.8$ vs. $21.4 \pm 4.1$; and VAP: $26.3 \pm 4.1$ vs. $26.8 \pm 4.4 \mu \mathrm{m} / \mathrm{s})$. For VAP and VSL, swim-up presented higher values $(P<0.05 ; 33.9 \pm 4.9$ vs. $29.4 \pm 4.7 \mu \mathrm{m} / \mathrm{s})$ than the other treatments, but was similar $(P>0.05)$ to mini-Percoll, Percoll and control group for VCL (swim-up: $44.0 \pm 5.2$; mini-Percoll: $40.1 \pm 4.6$; Percoll: $39.1 \pm 5.1$; control group: 40.8 $\pm 4.4 \mu \mathrm{m} / \mathrm{s})$. Control group presented the highest value for ALH $(1.8 \pm 0.1 \mu \mathrm{m})$ and it was not different from swim-up $(1.7 \pm 0.1 \mu \mathrm{m})$, that was similar $(P>0.05)$ to Percoll and mini-Percoll. For BCF, swim-up value was higher $(P<0.05 ; 6.6 \pm 0.4 \mathrm{~Hz})$ than the others. LIN and WOB values for swim-up technique were higher $(P<0.05 ; 52.9 \pm 4.6$ vs. $64.9 \pm 4.0 \%)$ than the other treatments. There was no difference $(P>0.05)$ between Percoll $(39.2 \pm 4.1$ vs. $54.5 \pm 3.8 \%)$ and mini-Percoll $(38.0 \pm 4.2$ vs. $54.4 \pm 3.7 \%)$ for these parameters, and values were higher $(P<0.05)$ than sperm washing $(31.4 \pm 4.2$ vs. $47.0 \pm 3.9 \%)$ and control group (29.0 \pm 3.2 vs. $47.8 \pm 2.9 \%)$, that were similar $(P>0.05)$ between each other.

Regarding to capacitation status immediately after the selection $(0 \mathrm{~h})$, control group and sperm washing by centrifugation obtained more $(P<0.05)$ capacitated cells, and swim-up and mini-Percoll recovered less $(P<0.05)$ cells. Control group and sperm washing recovered the lowest $(P<0.05)$ percentages of acrosome-reacted cells, whereas swim-up obtained the highest $(P<0.05)$. When we studied the PM integrity at $0 \mathrm{~h}$, intact cell rate was higher $(P<0.05)$ after swimup technique $(P<0.05)$. There was no difference $(P$ $>0.05)$ among the other treatments for PM integrity (Figure 1).

Overall, when we pool the data regardless of the interval of incubation (average from 0 to $3 \mathrm{~h}$ ), there was no difference $(P>0.05)$ for sperm washing, Percoll, mini-Percoll and swim-up, respectively, for: capacitated $(41.8 \pm 2.0,35.6 \pm 2.3,33.9 \pm 2.1$ and $30.3 \pm 2.4 \%)$ and noncapacitated $(5.3 \pm 1.0,4.6 \pm 0.8$, $5,5 \pm 1.1$ and $4.9 \pm 0.8 \%)$. When the sperm selections 
were compared with the control group, the last was higher $(P<0.05 ; 46.8 \pm 2.3 \%)$, than all treatments for capacitated. Regarding to acrosome-reacted rate, Percoll, mini-Percoll and swim-up had highest rates $(P<0.05 ; 59.8 \pm 2.4,60.6 \pm 2.3$ and $57.5 \pm 2.6 \%$, respectively), but swim-up was no different $(P>0.05)$ of sperm washing $(53.6 \pm 2.4 \%)$. Control group rate was lower $(P<0.05 ; 48.6 \pm 2.6 \%)$ than all treatments and was similar $(P>0.05)$ to sperm washing.

Regarding to PM integrity, when we compared the intervals of incubation among the treatments, swimup obtained higher $(P<0.05)$ rates of intact cells at $2 \mathrm{~h}$ of incubation (control group: $4.5 \pm 0.7$; sperm washing:
$4.2 \pm$ 0.5; Percoll: $5.0 \pm 1.7$; mini-Percoll: $7.4 \pm 2.2$; swim-up: $13.3 \pm 3.7 \%)$. On the other hand, there was no difference $(P>0.05)$ among the incubation intervals after each selection technique.

When we pool the data regardless of the interval of incubation (average from 0 to $3 \mathrm{~h}$ ), the intact cell rate was higher $(P<0.05)$ at swim-up $(17.4 \pm$ $2.8 \%)$. No differences $(P>0.05)$ were found among all the other treatments for either intact (Control: $8.0 \pm$ 1.7; sperm washing: $7.1 \pm 1.0$; Percoll: $9.4 \pm 1.8$; and mini-Percoll: $7.3 \pm 1.1 \%$ ) or damaged (Control: 91.9 \pm 1.7 ; sperm washing: $92.8 \pm 1.0$; Percoll: $90.6 \pm 1.8$; and mini-Percoll: $92.7 \pm 1.1 \%$ ) cells.

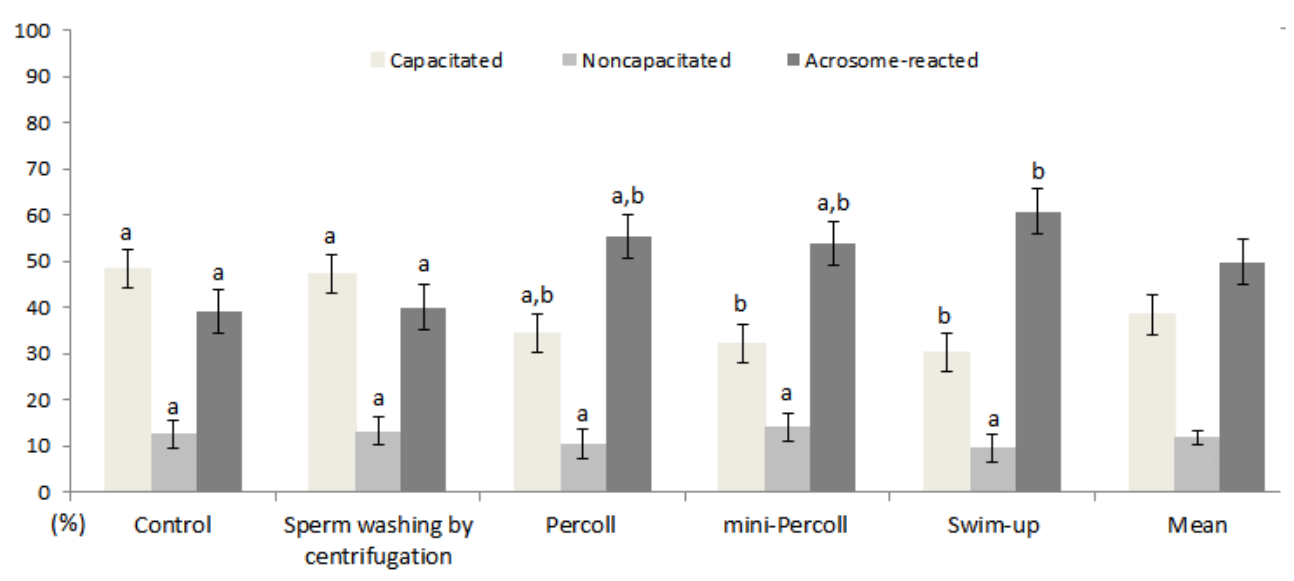

Intact $\quad$ Damaged

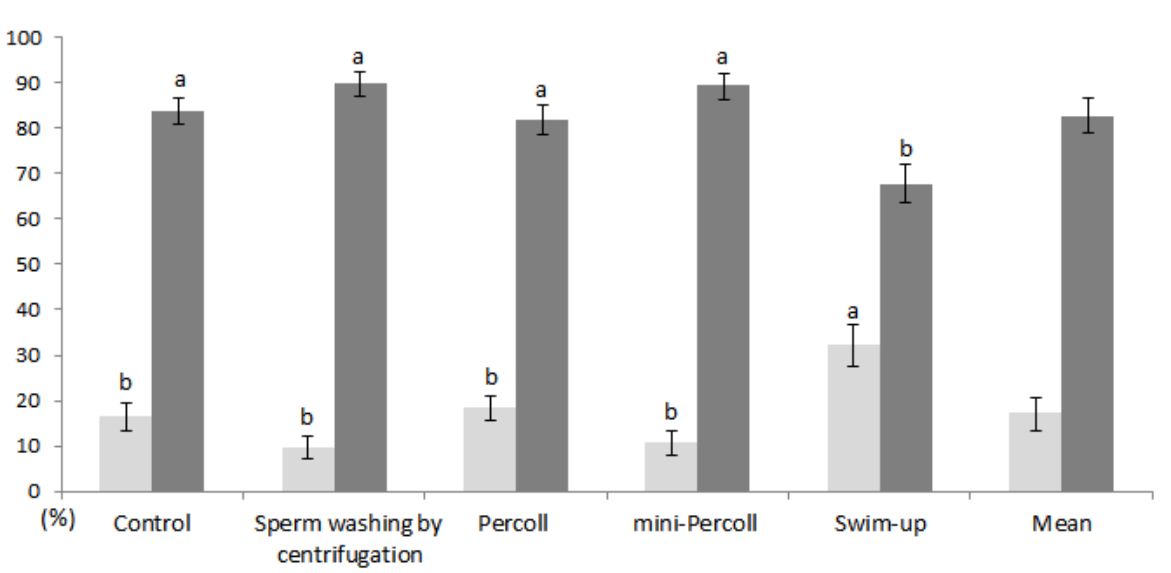

Figure 1. Capacitation status and plasma membrane (PM) integrity immediately after spermatozoa selection by different treatments in ram frozen-thawed sperm of Santa Inês breed (Mean \pm SEM). Within a column, values without a common superscript $(\mathrm{a}, \mathrm{b})$ differ significantly $(P<0.05) ; \mathrm{n}=10$. 


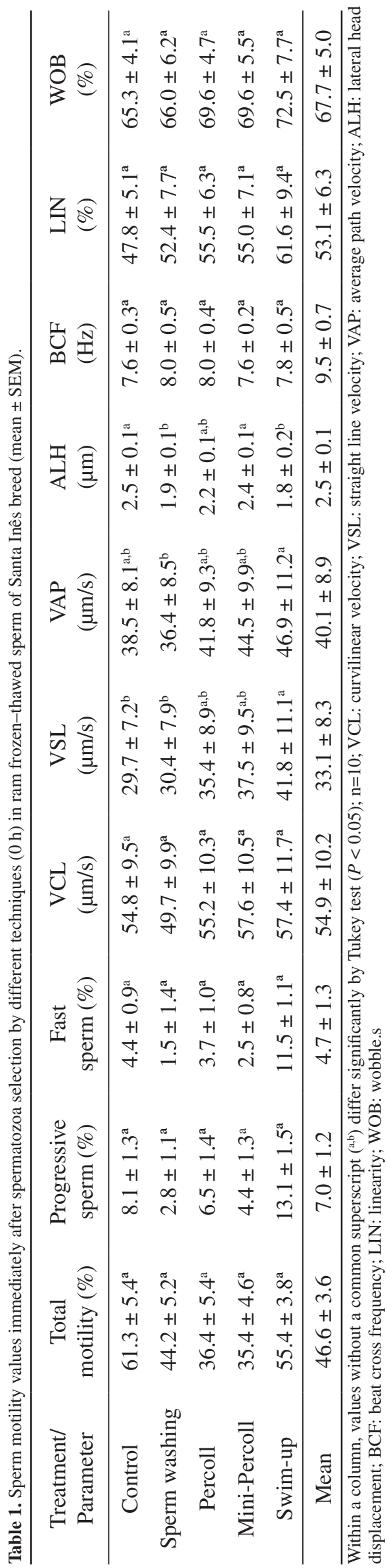

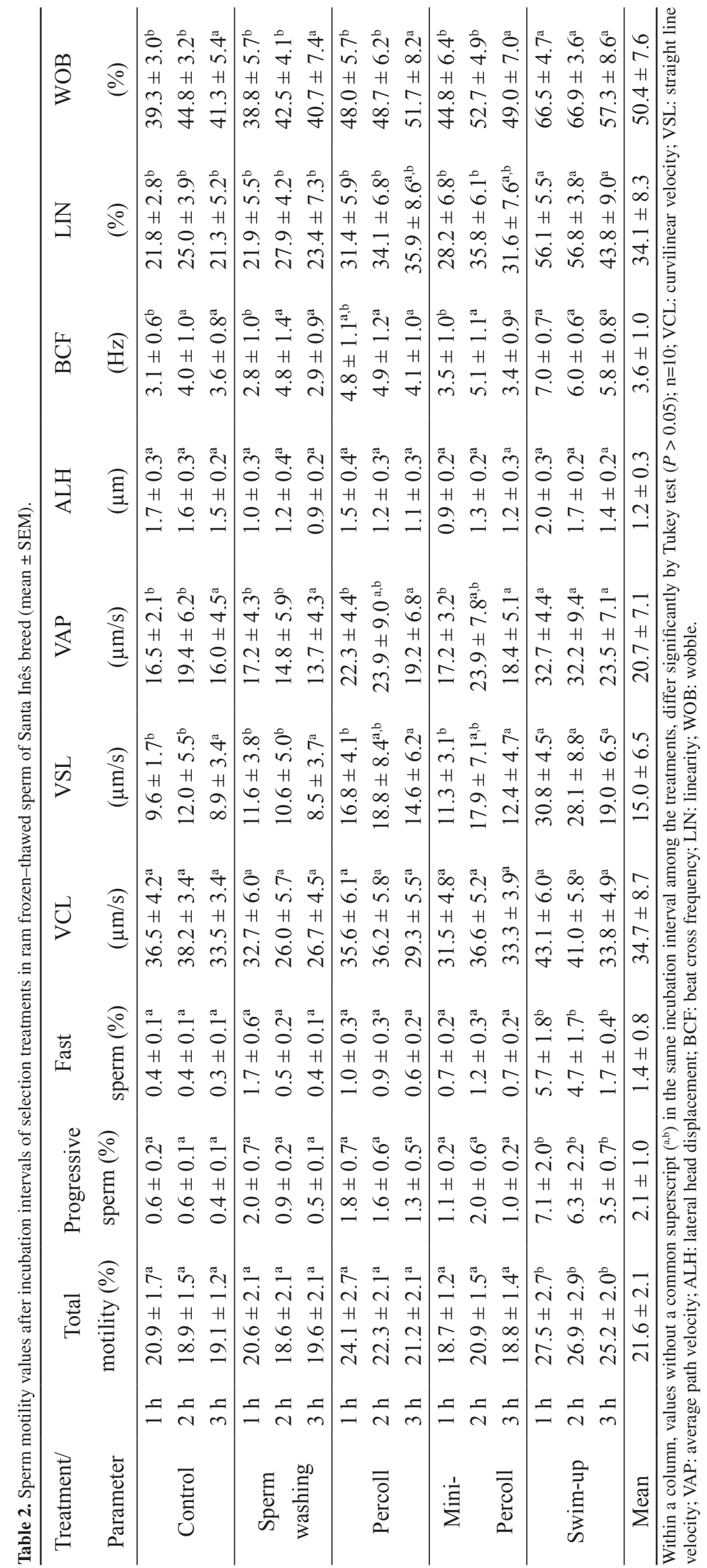




\section{DISCUSSION}

Different sperm selection techniques have been developed to obtain a procedure efficient to produce a final sample with great sperm parameters so fertilization may occur successfully. The sperm physiology and biochemistry are complex and a lot of studies are designed to evaluate the sperm cell and its preparation for receiving the oocyte under optimal conditions. Although the swim-up technique promoted higher rates for some of the sperm parameters evaluated, Percoll protocols are the most widely used procedures for selection during the sperm preparation for in vitro fertilization (IVF) in many species, possibly because of its greater speed, practical method and convenience compared to the swim-up technique.

Lower recovery rate was obtained after swimup, which is relatively low and corroborate with several other studies comparing different sperm selection techniques on bovine, ovine and caprine [24,27,30,36,37]. In this study, Percoll and mini-Percoll recovered approximately 10 times more cells than swim-up, which is an important feature to be considered during sperm preparation for IVF, being possible to use only one semen straw. The swim-up technique is based on the ability of mobile sperm migrate to the upper levels of the culture media, in a determinate interval [12]. On the other hand, the swim-down techniques, such as washing by centrifugation and density gradient methods, are based on the recovery of sperm by forced migration to the bottom of the tube, which is assigned to the gravitational force during centrifugation [36], which explains the difference between recovery rates among the sperm selection techniques obtained in this study.

Capacitated cells and acrosome-reacted rates were higher after each selection technique, when compared to the control group. These observations corroborate with Martí et al. [19] who reported that sperm selected by Percoll demonstrated greater hyperactivation ability and thus, greater ability to undergo capacitation, when compared to nonselected cells. The processing of the selection techniques is more time consuming and involves more than one centrifugation. The centrifugation processes generate mechanical stress, favoring damage to sperm cells and their PM during the selection techniques [7]. This fact probably holds up the destabilization of sperm PM, facilitating the occurrence of capacitation events and acrosome reaction. Furthermore, it is known that oxidative stress caused by the presence of reactive oxygen species (ROS) increased during centrifugations [1]. The presence of ROS induce lipid peroxidation causing considerable PM damage [22]. Beyond the stress of the centrifugation, extended incubation intervals, as in swim-up procedure, generate further oxidative stress [6]. Lastly, the incubation interval of the technique tends to make the incubated spermatozoa more sensitive to damage caused by the centrifugation [37], which explains the PM greater sensitivity of the selected sperm and occurrence of capacitation and acrosome reaction.

García-Álvarez et al. [9] observed that both capacitation or noncapacitation media in PT moment and after incubation showed high rates of PM fluidity in ram frozen-thawed sperm. In this same study, cells subjected to noncapacitation media underwent premature acrosome reaction. The authors attributed these facts to the cryopreservation process and described that after this process, the sperm present defects in $\mathrm{Ca}^{+2}$ regulation, being more susceptible to PM destabilization and capacitation. Similarly, the high capacitated and acrosome reaction rates observed after treatments in the current study are probably reinforced by changes in sperm cells caused by the cryopreservation process.

A low percentage of noncapacitated cells was observed at $0 \mathrm{~h}$ in the present study. When incubated, these cells seem to start the capacitation process immediately, and after $1 \mathrm{~h}$ this rate decreased drastically. The continuing decreased of this pattern can be explained due to the capacitation event and consequent acrosome reaction, which makes the rate of noncapacitated to greatly reduce after $3 \mathrm{~h}$ of incubation and the capacitated and acrosome-reacted rates high. In a similar study, after selection of frozen-thawed sperm by Percoll, Gillan et al. [10] found approximately $8 \%$ of noncapacitated cells. In addition, after $6 \mathrm{~h}$ incubation, noncapacitated cells were not found, and they found approximately $38 \%$ of capacitated cells and $68 \%$ of acrosome-reacted cells.

In order to strength the evidence that frozenthawed sperm, even after selection, is sensible and reactive to capacitation-like events, we demonstrated the capacitated and acrosome-reacted cell values immediately after the selection treatments behaved differently than when authors used ram fresh sperm [19]. The authors observed low capacitated and acrosome-reacted rates after swim-up $(12.5 \pm 4.2 \%$ and $15.2 \pm 3.3 \%)$. This difference can be due to cryocapacitation event that frozen sperm suffers, and thus is not observed in fresh semen [14]. Possibly, 
this capacitation-like changes observed in frozen-thawed sperm occurs regardless of the selection treatment used.

The capacitated rate remained constant up to $2 \mathrm{~h}$ and declined after $3 \mathrm{~h}$, which coincided with the increase of the acrosome-reacted rate. This behavior may suggest that the capacitation event in ram is time dependent and lasts around $2 \mathrm{~h}$ [2], once the sperm remained capacitated to $2 \mathrm{~h}$, initiating the process of acrosome reaction. These results corroborate with Pérez et al. [28]. The authors demonstrated, also using CTC fluorescence, that the ram frozen-thawed sperm underwent capacitation (90\%) faster than fresh sperm after $2 \mathrm{~h}$ of incubation.

Analyzing the motility parameters immediately after the selection, all treatments maintained or increased the rates compared to the control group. The swim-up, mini-Percoll and Percoll did not differ in any parameters. These results are important since the processing of selection techniques did not infer damages to spermatozoa. The sperm recovered by swim-up are considered excellent about biochemical, kinematics, motility and morphological patterns [12,35]. Given that Percoll and mini-Percoll did not show differences in relation to swim-up for motility parameters, such techniques can be used to replace the latter, obtaining similar sperm samples with good quality.

Regarding ALH immediately after selection, swim-up and sperm washing by centrifugation obtained samples with low rates. Holt and Palomo [13] observed this same behavior after ram sperm selection by swim-up. The authors suggest that this is due to a cleaner culture media promoted by the technique, free of debris and cytoplasmic droplets, improving route and sperm quality. This result is positive since high ALH values are associated with low quality sperm that moves in aleatory tracks with low linearity and progressivity, which is unwanted [13].

The VSL parameter was the only velocity that got improvement in relation to control group, and just after swim-up. Holt and Palomo [13] affirmed VSL is the parameter that most strongly characterizes the quality of ram selected sperm. However, immediately after treatments and regardless of the incubation interval, the selected sperm by swim-up, showed higher values for VSL and also for VAP, corroborating with other studies $[7,13]$. In ovine, there are still some discrepancies in relation to the motility parameters that can be well correlated with fertility. Robayo et al. [31] reported VAP was positively correlated with sperm migration in the cervical mucus, and thus, a parameter more strongly correlated with fertility.

Swim-up technique, after incubation, also showed better results for several sperm parameters, including LIN, enhancing that the incubated cells recovered by this technique presents better quality [12]. According to García-Álvarez et al. [8], high values of LIN indicate sperm motility patterns sperm related with most progressive sperm and acquisition of fertilizing ability.

When assessing the motility parameters after treatment and incubation, swim-up showed superiority in relation to total progressive and fast cell percentages. These results are consistent with other studies that also found superiority in various motility parameters after swim-up [13,20]. The fact the swim-up technique select more mobile cells which feature progressive movement and ability to reach the top of the media [27], explains the greater amount of such subpopulations in the final sample.

Regarding to PM integrity of selected and incubated sperm, the only treatment that was superior to the control and the others was swim-up, corroborating with other studies in buffalos [20], bucks [25] and bulls [27]. Swim-up involves a procedure that recovers a clean fraction without debris and other types of cells, with high rate of mobile sperm with excellent quality [35], reason why it can justify the higher recovery of intact spermatozoa after the technique.

\section{CONCLUSIONS}

Spermatozoa selected by swim-up presents higher sperm parameters values throughout the incubation intervals, indicating a more long-lived sample and an advantage for IVF. However, Percoll protocols recover 10 times more cells than the swim-up, suggesting these protocols represent a great economical advantage for ram frozen-thawed sperm selection. Moreover, Percoll and mini-Percoll techniques did not show sperm injuries in comparison with the control group, thus, not affecting sperm cells along time.

\section{MANUFACTURERS}

${ }^{1}$ Sigma Chemical. St. Louis, MO, USA.

${ }^{2}$ Halotech DNA. Madrid, Spain.

${ }^{3}$ Eppendorf Brasil. São Paulo, SP, Brazil.

${ }^{4}$ Corning Incorporated. São Paulo, SP, Brazil.

${ }^{5}$ Nikon Corporation. Tokyo, Japan.

${ }^{6}$ Automatic Diagnostic Systems. Barcelona, Spain.

${ }^{7}$ Viçosa Federal University. Viçosa, MG, Brazil. 
Acknowledgments. This research was supported by Finep and Faperj. JFF, FZB and CCSO were supported by CNPq and JMGSF, by CAPES.

Funding. The research reported was funded by different Brazilian Institutes that were all cited in the Acknowledgments: Finep and Faperj (E-26/111.694/2013).
Ethical approval. All procedures and treatments care were in compliance with Ethics Committee in the use of animals of Fluminense Federal University (protocol approval: 374/13).

Declaration of interest. This manuscript has never been published. The authors are in agreement with statements and requirements for publication. None of the authors have any conflict of interest to declare.

\section{REFERENCES}

1 Aitken R.J. \& Clarson J.S. 1988. Significance of reactive oxygen species and antioxidants in defining the efficacy of sperm preparation techniques. Journal of Andrology. 9: 367-376.

2 Austin C.R. 1970. Ageing and reproduction: post-ovulatory deterioration of the egg. Journal of Reproduction and Fertility (Suppl). 12: 39-53.

3 Chamberland A., Fournier I.V., Tardff S., Sirard I.M.A., Sulhvan I.R. \& Badey J.L. 2001. The effect of heparin on motility parameters and protein phosphorylation during bovine sperm capacitation. Theriogenology. 55: 823-835.

4 Cocero M.J., Alabart J.L., Hammami S., Martí J.I., Lahoz B., Sánchez P., Echegoyen E., Beckers J.F. \& Folch J. 2011. The efficiency of in vitro ovine embryo production using an undefined or a defined maturation medium is determined by the source of the oocyte. Reproduction in Domestic Animals. 46: 463-470.

5 Cormier N., Sirard M.A. \& Bailey J.L. 1997. Premature Capacitation of Bovine Spermatozoa Is Initiated by Cryopreservation. Journal of Andrology. 18: 461-468.

6 Correa J.R. \& Zavos P.M. 1996. Preparation and recovery of frozen-thawed bovine spermatozoa via various sperm selection techniques employed in assisted reproductive technologies. Theriogenology. 46: 1225-1232.

7 García-Álvarez O., Maroto-Morales A., Ramón M., Del Olmo E., Montoro V., Dominguez-Rebolledo A.E., Bisbal A., Jiménez-Rabadán P., Pérez-Guzmán M.D. \& Soler A.J. 2010. Analysis of selected sperm by density gradient centrifugation might aid in the estimation of in vivo fertility of thawed ram spermatozoa. Theriogenology. 74: 979-988.

8 García-Álvarez O., Maroto-Morales A., Ramón M., Del Olmo E., Jiménez-Rabadán P., Fernández-Santos M.R., Anel-López L., Garde J.J. \& Soler A.J. 2014. Dynamics of sperm subpopulations based on motility and plasma membrane status in thawed ram spermatozoa incubated under conditions that support in vitro capacitation and fertilization. Reproduction, Fertilily and Development. 26: 725-732.

9 García-Álvarez O., Maroto-Morales A., Jiménez-Rabadán P., Ramón M., Del Olmo E., Iniesta-Cuerda M., AnelLópez L., Fernández-Santos M.R., Garde J.J. \& Soler A.J. 2015. Effect of different media additives on capacitation of frozen-thawed ram spermatozoa as a potential replacement for estrous sheep serum. Theriogenology. 84: 948-955.

10 Gillan L., Evans A.G. \& Maxwell W.M.C. 1997. Capacitation status and fertility of fresh and frozen-thawed ram spermatozoa. Reproduction, Fertility and Development. 9: 481-487.

11 Henkel R.R. 2012. Sperm preparation: state-of-the-art-physiological aspects and application of advanced sperm preparation methods. Asian Journal of Andrology. 14: 260-269.

12 Henkel R.R. \& Schill W.B. 2003. Sperm preparation for ART. Reproductive Biology and Endocrinology. 1: 108-129.

13 Holt W.V. \& Palomo M.J. 1996. Optimization of a continuous real-time computerized semen analysis system for ram sperm motility assessment, and evaluation of four methods of semen preparation. Reproduction, Fertility and Development. 8: 219-230.

14 Kadirvel G., Kumar S., Kumaresan A. \& Kathiravan P. 2009. Capacitation status of fresh and frozen-thawed buffalo spermatozoa in relation to cholesterol level, membrane fluidity and intracellular calcium. Animal Reproduction Science. 116: 244-253.

15 Luna C., Colás C., Casao A., Serrano E., Domingo J., Pérez-Pé R., Cebrián-Pérez J.A. \& Muiño-Blanco T. 2015. Ram seminal plasma proteins contribute to sperm capacitation and modulate sperm-zona pellucida interaction. Theriogenology. 83: 670-678.

16 Machado G.M., Carvalho J.O., Siqueira Filho E., Caixeta E.S., Franco M.M., Rumpf R. \& Dode M.A.N. 2009. Effect of Percoll volume, duration and force of centrifugation, on in vitro production and sex ratio of bovine embryos. Theriogenology. 71: 1289-1297. 
17 Maia M.S., Bicudo S.D., Azevedo H.C., Sicherle C.C., De Sousa D.B. \& Rodello L. 2009. Motility and viability of ram sperm cryopreserved in a Tris-egg yolk extender supplemented with anti-oxidants. Small Ruminant Research. 85: 85-90.

18 Mara L., Sanna D., Casu S., Dattena M. \& Muñoz I.M.M. 2013. Blastocyst rate of in vitro embryo production in sheep is affected by season. Zygote. 22: 366-371.

19 Martí E., Pérez-Pé R., Muiño-Blanco T. \& Cebrián-Pérez J.A. 2006. Comparative study of four different sperm washing methods using apoptotic markers in ram spermatozoa. Journal of Andrology. 27: 746-753.

20 Mehmood A., Anwar M. \& Naqvi S.M.S. 2009. Motility, acrosome integrity, membrane integrity and oocyte cleavage rate of sperm separated by swim-up or Percoll gradient method from frozen-thawed buffalo semen. Animal Reproduction Science. 111: 141-148.

21 Morrell J.M. \& Rodríguez-Martínez H. 2009. Biomimetic techniques for improving sperm quality in animal breeding: a review. Open Andrology Journal. 1: 1-9.

22 Mortimer D. 1991. Sperm preparation techniques and iatrogenic failures of in vitro fertilization. Human Reproduction. 6: 173-176.

23 Morton K., Maxwell W. \& Evans G. 2008. Effect of aspiration pressure during oocyte harvesting on oocyte recovery and in vitro development of ovine oocytes. Reproduction in Domestic Animals. 43: 106-110.

24 Olivares C.C.S., Fonseca J.F., Camargo L.S.A., Souza-Fabjan J.M.G., Rodrigues A.L.R. \& Brandão F.Z. 2015. Comparison of different methods of goat sperm selection and capacitation for optimization of assisted reproductive technologies. Small Ruminant Research. 127: 44-49.

25 Palomo M.J., Izquierdo D., Mogas T. \& Paramio M.T. 1999. Effect of semen preparation on IVF of prepubertal goat oocytes. Theriogenology. 51: 927-940.

26 Papadopoulos S., Hanrahan J.P., Donovan A., Duffy P., Boland M.P. \& Lonergan P. 2005. In vitro fertilization as a predictor of fertility from cervical insemination of sheep. Theriogenology. 63: 150-159.

27 Parrish J.J., Krogenaes A. \& Susko-parrish J.L. 1995. Effect of bovine sperm separation by either Swim-up or percoll method on success of in vitro fertilization and early embryonic development. Theriogenology. 44: 859-869.

28 Pérez L.J., Valcarcel A., Heras M.A., Moses D. \& Baldasarre H. 1996. Evidence that frozen/thawed ram spermatozoa show accelerated capacitation in vitro as assessed by Chlortetracycline assay. Theriogenology. 46: 131-140.

29 Pérez-Pé R., Grasa P., Fernández-Juan M., Peleato M.L., Cebrián-Pérez J.A. \& Muiño-Blanco T. 2002. Seminal plasma proteins reduce protein tyrosine phosphorylation in the plasma membrane of cold-shocked ram spermatozoa. Molecular Reproduction and Development. 61: 226-233.

30 Rho G.J., Hahnel A.C. \& Betteridge K.J. 2001. Comparisons of oocyte maturation times and of three methods of sperm preparation for their effects on the production of goat embryos in vitro. Theriogenology. 56: 503-516.

31 Robayo I., Montenegro V., Valdés C. \& Cox J. 2008. CASA Assessment of kinematic parameters of ram spermatozoa and their relationship to migration efficiency in ruminant cervical mucus. Reproduction in Domestic Animals. 43: 393-399.

32 Rodríguez-Martínez H. 2006. Can we increase the estimative value of semen assessment? Reproduction in Domestic Animals. 41: 2-10.

33 Said T., Agarwal A., Grunewald S., Rasch M., Baumann T., Kriegel C., Li L., Glander H., Thomas A.J. \& Paasch U. 2006. Selection of nonapoptotic spermatozoa as a new tool for enhancing assisted reproduction outcomes: An in vitro model. Biology of Reproduction. 74: 530-537.

34 Shirazi A. \& Motaghi E. 2013. The in vitro fertilization of ovine oocytes in the presence of oviductal cells and its effect on the expression of zygote arrest 1 (zar1) and subsequent embryonic development. Journal of Reproduction and Infertility.14: 8-16.

35 Shojaei H., Kroetsch T., Wilde R., Blondin P., Kastelic J.P. \& Thundathil J.C. 2012. Moribund sperm in frozenthawed semen, and sperm motion end points post-thaw and post-swim-up, are related to fertility in Holstein AI bulls. Theriogenology. 77: 940-951.

36 Shokrollahi E., Barati F. \& Gooraninejad S. 2014. Efficacy of Histoprep ${ }^{\circledR}$ gradient for isolating ovine epididymal sperm. Small Ruminant Research. 119: 96-99.

37 Somfai T. 2002. Effect of swim up and percoll treatment on viability and acrossome integrity of frozen-thawed bull spermatozoa. Reproduction in Domestic Animals. 37: 285-290. 
C.C.S. Olivares, J.M.G. Souza-Fabjan, J.F. Fonseca, et al. 2017. Comparison of Different Sperm Selection Techniques in Ram Frozen-Thawed Sperm. Acta Scientiae Veterinariae. 45: 1431.

38 Wang Z., Lin P. \& Yu S. 2013. Effects of ghrelin on developmental competence and gene expression of in vitro fertilized ovine embryos. Theriogenology. 79: 695-701.

39 Zúccari C.E.S.N., Carrijo P.R., Leite P.A., Scaldelai P.R.R., Rodovalho N.C.M., Zanenga C.A., Kiefer C. \& Silva E.V.C. 2008. Seleção em gradiente de Percoll sobre os parâmetros espermáticos do sêmen bovino congelado. Revista Brasileira de Saúde e Produção Animal. 9: 358-366. 